\title{
Covariant form of the ideal magnetohydrodynamic "connection theorem" in a relativistic plasma
}

F. Pegoraro

Dipartimento di Fisica "Enrico Fermi", Università di Pisa, Largo B. Pontecorvo 3, I-56127 Pisa, Italy

PACS 52.30. Cv - Magnetohydrodynamics

PACS 52.27.Ny - Relativistic plasmas

PACS 95.30.Qd - Magnetohydrodynamics and plasmas

\begin{abstract}
The magnetic connection theorem of ideal Magnetohydrodynamics by Newcomb [Newcomb W.A., Ann. Phys., 3 , 347 (1958)] and its covariant formulation are rederived and reinterpreted in terms of a "time resetting" projection that accounts for the loss of simultaneity in different reference frames between spatially separated events.
\end{abstract}

Introduction. - The dynamics of relativistic plasmas is presently under extensive investigation, both in the context of laboratory plasmas such as laser produced plasmas and in the context of high energy astrophysics, and the concept of relativistic magnetic reconnection is frequently used in the literature (see e.g., Ref. 1]).

However, the meaning of "magnetic connection" (i.e., of the property that should be broken by the "reconnection processes" leading to the release of magnetic energy), and the concept of magnetic field line motion and, in fact, of magnetic topology itself may not be evident in the case of a relativistic plasma dynamics where the distinction between electric and magnetic fields is frame dependent. This is in particular the case in the presence of large, inhomogeneous velocity fields where it is not clear how to identify a preferred reference frame.

Magnetic connections of fluid plasma elements. Within the Magnetohydrodynamic (MHD) description of a nonrelativistic plasma, the ideal Ohm's law $\vec{E}+\vec{v} \times \vec{B}=0$ allows us to give a meaning to the concept of motion of a magnetic field line.

In fact it can be shown 2] that if two plasma elements initially located at positions $\vec{x}_{1}$ and $\vec{x}_{2}$ are connected by a magnetic line, and if they move with the (fluid) plasma velocity $\vec{v}(\vec{x}, t)$ that satisfies the ideal Ohm's law, then for every following time there will be a magnetic line that connects them. Here $\vec{B}(\vec{x}, t)$ and $\vec{E}(\vec{x}, t)$ are the magnetic and electric fields in the plasma.

The proof of this statement does not require the use of the "inhomogeneous" Maxwell's equations, i.e. of the
Maxwell's equations that relate the electromagnetic fields to their sources. Thus it does not depend on the assumptions that are made in nonrelativistic MHD, such as quasineutrality and the neglecting of the displacement current that cannot be applied to a relativistic MHD plasma description 3. In other words, provided the ideal Ohm's law is satisfied, the equation for the magnetic field written in three-dimensional (3D) notation

$$
\partial \vec{B} / \partial t-\nabla \times(\vec{v} \times \vec{B})=0
$$

is valid independently of the plasma being relativistic or not, although clearly it is not explicitly covariant.

With appropriate assumptions on the smoothness of the velocity field the "connection theorem" can be proved by computing the Lagrangian derivative along the fluid trajectories of the expression $\delta \vec{l} \times \vec{B}$ where $\delta \vec{l}$ is the $3 \mathrm{D}$ vector connecting to infinitesimally close fluid elements. We obtain

$$
\begin{gathered}
\frac{d}{d t} \vec{B}=\vec{\nabla} \times(\vec{v} \times \vec{B})+(\vec{v} \cdot \vec{\nabla}) \vec{B}, \\
\frac{d}{d t} \delta \vec{l}=(\delta \vec{l} \cdot \vec{\nabla}) \vec{v},
\end{gathered}
$$

and finally,

$$
\begin{aligned}
& \frac{d}{d t}(\delta \vec{l} \times \vec{B})=-(\delta \vec{l} \times \vec{B})(\vec{\nabla} \cdot \vec{v}) \\
& -[(\delta \vec{l} \times \vec{B}) \times \vec{\nabla}] \times \vec{v},
\end{aligned}
$$

which ensures that if a $t=0$ the $\delta \vec{l}$ separation vector is parallel to $\vec{B}$, i.e. if $[\overrightarrow{b l} \times \vec{B}]_{t=0}=0$, then it remains zero at 
all times. As for Eq.(11), Eqs.(2] (3) are valid independently of the plasma being relativistic or not, although they are not explicitly covariant.

A relativistically covariant generalization of the concept of magnetic topology (see e.g. the extended analysis presented in Ref. 4) encounters two major related obstacles: the loss of the concept of simultaneity between spatially separated events in different reference frames and the fact that the electric and the magnetic fields are not independent quantities but are the components of an antisymmetric tensor that transforms in different frames under appropriate Lorentz transformations.

It is possible however, in line with the results presented in Ref. 2] but with a different perspective, to rewrite Eqs.(14) in a fully covariant form and to generalize in a frame independent way the concept of magnetic connection to a relativistic plasma within the ideal MHD description.

Covariant Lagrangian field equation. - Using standard relativistic notation with Greek indices running from 0 to 3 and the summation convention over dummy indices, the ideal Ohm's law in four dimensional (4D) covariant notation takes the form

$$
F_{\mu \nu} u_{\mu}=0
$$

where $F_{\mu \nu}\left(x_{\alpha}\right)$ is the electromagnetic field tensor [5, $x_{\alpha}$ is the position four vector and $u_{\alpha}=d x_{\alpha} / d \tau$ the four velocity of the fluid element with $\tau$ its proper time.

Equation (5) can be rewritten as a covariant equation for the variation of the four vector potential $A_{\nu}$ along the fluid motion in the (Lagrangian) form

$$
d A_{\nu} / d \tau=u_{\mu}\left(\partial_{\nu} A_{\mu}\right)
$$

where $d / d \tau=u_{\mu} \partial_{\mu}$ has been used with $\partial_{\mu} \equiv \partial / \partial x_{\mu}$. By computing the Lagrangian derivative of the electromagnetic field tensor with respect to the proper time $\tau$, using Eq.(6) and recalling that $\partial_{\mu}(d / d \tau \ldots) \neq d / d \tau\left(\partial_{\mu} \ldots\right)$, we obtain

$$
d F_{\mu \nu} / d \tau=\left(\partial_{\mu} u_{\alpha}\right) F_{\nu \alpha}-\left(\partial_{\nu} u_{\alpha}\right) F_{\mu \alpha},
$$

which is the covariant counterpart of Eq.(1) or, more exactly, of its Lagrangian form given by Eq.(2).

In a given frame, projecting Eq.(7) onto its space-space and space-time components and writing $\left(\partial_{\mu} u_{\alpha}\right) F_{\nu \alpha}=$ $\gamma F_{\nu \alpha} \partial_{\mu}\left(u_{\alpha} / \gamma\right)$ with $\gamma$ the Lorentz factor (where we use $\left.F_{\alpha \nu} u_{\alpha}=0\right)$ we recover Eq.(2) for the magnetic field, in terms of the Lagrangian time derivative $d / d t=\partial / \partial t+\vec{v} \cdot \nabla$, plus the associated equation for the Lagrangian time derivative of the electric field $\vec{E}$.

Covariant connection equation. - Let us now introduce the spacelike event-separation four vector $d l_{\mu}$ and refer first to the frame where $d l_{0}=0$, i.e., where the two events are simultaneous. In this frame the condition $F_{\mu \nu} d l_{\mu}=0$ is equivalent to $d \vec{l} \times \vec{B}=0$ and includes the additional condition $d \vec{l} \cdot \vec{E}=0$ that follows from the ideal Ohm's law if $d \vec{l} \times \vec{B}=0$.

Note that the condition $F_{\mu \nu} d l_{\mu}=0$ requires that the Lorentz invariant $F_{\mu \nu} F_{\mu \nu}^{*}$ vanishes (i.e. $\vec{E} \cdot \vec{B}=0$ ). Here $F_{\mu \nu}^{*} \equiv \varepsilon_{\mu \nu \alpha \beta} F_{\alpha \beta} / 2$ is the dual tensor of $F_{\mu \nu}$ and $\varepsilon_{\mu \nu \alpha \beta}$ is the completely antisymmetric Ricci tensor in $4 \mathrm{D}$. Because of the ideal Ohm's law (5), this condition is satisfied.

Then, independently of the frame chosen, $d l_{\mu}$ belongs to a $2 \mathrm{D}$ hyperplane. If the Lorentz invariant $F_{\mu \nu} F_{\mu \nu}$ is negative, i.e., if $E^{2}<B^{2}$ (which is the case we consider since $u_{\mu}$ is timelike), in this hyperplane we can choose one timelike (e.g., along $u_{\mu}$ ) and one spacelike direction (along $\left.d l_{\mu}\right)$.

Defining the $4 \mathrm{D}$ displacement of a plasma fluid element $\Delta x_{\mu}=u_{\mu} \Delta \tau$, we find

$$
\Delta d l_{\mu}=\left[d l_{\alpha}\left(\partial_{\alpha} u_{\mu}\right)\right] \Delta \tau+u_{\mu}\left[d l_{\alpha}\left(\partial_{\alpha} \Delta \tau\right)\right],
$$

that generalizes the corresponding 3D expression given by Eq.(3) and includes the coordinate dependence of the proper time variation.

Finally, from Eqs.(78), again using $F_{\mu \nu} u_{\mu}=0$, we obtain

$$
\frac{d}{d \tau}\left(d l_{\mu} F_{\mu \nu}\right)=-\left(\partial_{\nu} u_{\beta}\right)\left(d l_{\alpha} F_{\alpha \beta}\right) .
$$

which is the covariant form of Eq.(44).

Projection along the trajectories. - In a reference frame where $d l_{0} \neq 0$, i.e., where the two events are not simultaneous, we can "project" $d l_{\mu}$ onto $3 \mathrm{D}$ space along the fluid trajectories by defining $d l_{\mu}^{\prime}=d l_{\mu}-u_{\mu} d \lambda$, such that $d l_{0}^{\prime}=0$, without changing the connection equation since $F_{\mu \nu} u_{\mu}=0$. This shows that in order to recover the standard form of the connection theorem in terms of the magnetic field alone it is sufficient to reset the time by moving the endpoints of the word-line connecting the two close events along their trajectories. This result amounts to a rewording of the corresponding discussion given in Ref. 2].

Conclusions. - The magnetic connection theorem of ideal MHD can be cast in a covariant, frame independent, form but its interpretation in terms of magnetic field lines alone requires that the time of the two connected plasma elements be reset so as to restore simultaneity when the reference frame is changed.

This procedure is made possible by the assumption that the relativistic plasma dynamics obeys the ideal Ohm's law that allows us to move the endpoints of the word-line connecting the two close events along their trajectories without changing the connection equation.

Having established a covariant formulation of the connection theorem it will now be possible to reconsider in a covariant framework the related topological properties of an ideal MHD plasma, such as in particular the so called "linking number" between closed magnetic field lines.

Independently of this topological formulation we note that Eq. (7) represents a convenient form for advecting the 
e.m. fields in an ideal MHD plasma in a frame independent way, while Eq. (9) can provide an accuracy test for Relativistic ideal-MHD numerical codes.

More importantly, the time resetting procedure obtained by a projection along the trajectories of the plasma elements should allow us to describe in a frame independent way the redistribution of the magnetic plasma configuration in the presence of a reconnection event, when the connections are locally broken in space and time as Eq.(1) is locally violated. Vice-versa it will allow us to locate in a frame independent way where in space and in time the reconnection event has occurred (see e.g., the point raised in Ref. [6]). As mentioned before, this can be important in the presence of large inhomogeneous velocity fields that prevent us from selecting a preferred reference frame.

The research leading to these results has received funding from the European Commission's Seventh Framework Programme (FP7/20072013) under the SWIFF grant agreement (project n 263340, www.swiff.eu).

\section{REFERENCES}

[1] Zenitani S., Hesse M. and Klimas A., Astrophys. J. Lett., 716 (2010) L214.

[2] Newcomb W.A., Ann. Phys., 3 (1958) 347.

[3] Lichnerowicz A., Relativistic Hydrodynamics and Magnetohydrodynamics, (A.S. Wightman, W.A. Benjamin Inc., New York) 1967.

[4] Hornig G., Phys. Plasmas, 4 (1997) 646.

[5] Landau L.D. and Lifschitz E.M., The Classical Theory of Fields (Butterworth - Heinemann, Oxford) 1975.

[6] Zenitani S., Hesse M., Klimas A. and Kuznetsova M., Phys. Rev. Lett., 106 (2011) 195003. 